PROCEEDINGS OF THE

AMERICAN MATHEMATICAL SOCIETY

Volume 134, Number 10, October 2006, Pages 2785-2794

S 0002-9939(06)08573-X

Article electronically published on May 4, 2006

\title{
A REMARK ON THE MONOMIAL CONJECTURE AND COHEN-MACAULAY CANONICAL MODULES
}

\author{
LE THANH NHAN
}

(Communicated by Bernd Ulrich)

\begin{abstract}
In this paper, some sufficient conditions for rings and modules to satisfy the monomial conjecture are given. A characterization of CohenMacaulay canonical modules is presented.
\end{abstract}

\section{INTRODUCTION}

Throughout this paper, $(R, \mathfrak{m})$ is a Noetherian local $\operatorname{ring}$ with $\operatorname{dim} R=d$ and $M$ a finitely generated $R$-module with $\operatorname{dim} M=d^{\prime}$. The monomial conjecture of Hochster [H1] asserts that for any given system of parameters (s.o.p. for short) $\underline{x}=\left(x_{1}, \ldots, x_{d}\right)$ of $R$,

$$
x_{1}^{t} \ldots x_{d}^{t} \notin\left(x_{1}^{t+1}, \ldots, x_{d}^{t+1}\right) R \text { for all } t>0 .
$$

The Monomial Conjecture (MC) has been proved for the equicharacteristic case; cf. [C, Proposition 3]. In the mixed and the positive characteristic cases, (MC) and the Direct Summand Conjecture are equivalent to the Canonical Element Conjecture [H2]. Therefore (MC) makes a central role in the study of these important homological conjectures. (MC) has also been proved when $\operatorname{dim} R \leqslant 2$ (cf. [H1]), and when $R$ is a Buchsbaum ring; cf. [Go, Corollary 4.8]. By using the theory of modules of generalized fractions, Sharp-Zakeri SZ2 proved some results related to (MC) for rings of dimension $d$ under the assumption that (MC) is valid for rings of dimension $d-1$. There is also a reduction of (MC) given by Strooker-Stückrad SS]: (MC) is valid for all local rings if and only if for any complete intersection ring $A$ and any ideal $\mathfrak{a}$ of $A$ consisting of zero divisors, $A_{n} n_{A} \mathfrak{a}$ is not contained in any parameter ideal of $A$. Recently, Dutta D] gave some nice sufficient conditions for (MC).

(MC) has also been raised for modules: An s.o.p. $\underline{x}=\left(x_{1}, \ldots, x_{d^{\prime}}\right)$ of $M$ is said to satisfy (MC) if $x_{1}^{t} \ldots x_{d^{\prime}}^{t} M \nsubseteq\left(x_{1}^{t+1}, \ldots, x_{d^{\prime}}^{t+1}\right) M$ for all $t>0$. Hochster [H1] showed that in general (MC) does not hold for every s.o.p. of $M$, but it holds for high powers of s.o.p., i.e. there exists for each s.o.p. $\underline{x}=\left(x_{1}, \ldots, x_{d^{\prime}}\right)$ of $M$ an integer $n_{\underline{x}}>0$ such that $\left(x_{1}^{n_{1}}, \ldots, x_{d^{\prime}}^{n_{d^{\prime}}}\right)$ satisfies $(\mathrm{MC})$ for all $n_{1}, \ldots, n_{d^{\prime}} \geq n_{\underline{x}}$. Then Cuong-Hoa-Loan [CHL, Th. 3.3] proved that there always exists an integer

Received by the editors January 11, 2005.

2000 Mathematics Subject Classification. Primary 13D45, $13 \mathrm{H} 10$.

Key words and phrases. Monomial conjecture, strict f-sequences, Cohen-Macaulay canonical modules.

The author is a junior associate member of ICTP, Trieste, Italy.

(C)2006 American Mathematical Society Reverts to public domain 28 years from publication 
$c>0$ such that $\left(x_{1}^{n_{1}}, \ldots, x_{d^{\prime}}^{n_{d^{\prime}}}\right)$ satisfies (MC) for all s.o.p. $\left(x_{1}, \ldots, x_{d^{\prime}}\right)$ of $M$ and all integers $n_{1}, \ldots, n_{d^{\prime}} \geq c$ (see also [St, Corollary 11.2.4], [D, Corollary 2] for the case $M=R$ ). In this case, $c$ is called a uniform bound for $(M C)$ of $M$.

The notion of Cohen-Macaulay canonical modules was introduced by Schenzel Sc]: $M$ is called Cohen-Macaulay canonical if its canonical module exists and is Cohen-Macaulay. Note that if $R$ has a Cohen-Macaulay canonical module of dimension $\operatorname{dim} R$, then $R$ has a maximal Cohen-Macaulay module and hence (MC) is valid for $R$. Therefore it is worth finding conditions for a module to be CohenMacaulay canonical.

The purpose of this paper is to use strict f-sequences introduced in [CMN] as a useful tool to study the Monomial Conjecture and characterize Cohen-Macaulay canonical modules.

This paper is divided into four sections. In the next section, we give a sufficient condition for an s.o.p. to satisfy (MC) (Theorem 2.3). As a consequence, we show for each strict f-sequence s.o.p. $\underline{x}=\left(x_{1}, \ldots, x_{d^{\prime}}\right)$ of $M$ a concrete integer $n_{\underline{x}}$ such that $\left(x_{1}, \ldots, x_{d^{\prime}-1}, x_{d^{\prime}}^{n}\right)$ satisfies (MC) when $n>n_{\underline{x}}$ (cf. Corollaries 2.4 and 2.6). Then we prove that Theorem 1.6 of Dutta $\mathrm{D}$ ] can be extended from rings to modules (Corollary 2.5). In Section 3, we study a stronger kind of uniform bound which is called a strong uniform bound for (MC): An integer $c>0$ is called a strong uniform bound for $(M C)$ of $M$ if $\left(x_{1}, \ldots, x_{d^{\prime}-1}, x_{d^{\prime}}^{n}\right)$ satisfies (MC) for all s.o.p. $\left(x_{1}, \ldots, x_{d^{\prime}}\right)$ of $M$ and all $n \geq c$. In the last section, we characterize Cohen-Macaulay canonical modules (Theorem 4.2). As a consequence, we give a sufficient condition for a ring to satisfy (MC), and again we get theorems of Schenzel $[\mathrm{Sc}$, Theorem 4.3, Theorem 4.6 (iii)] by elementary proofs.

\section{A sufficient condition for the Monomial Conjecture}

In this section we give a sufficient condition such that (MC) is satisfied. We first need the notion of a strict f-sequence introduced in [CMN]. For each Artinian module $A$, we denote by $\operatorname{Att} A$ the set of all attached primes of $A$; see $\mathrm{Mac}$.

Definition 2.1. A sequence $\left(x_{1}, \ldots, x_{k}\right)$ of elements in $\mathfrak{m}$ is called a strict $f$ sequence of $M$ if $x_{j+1} \notin \mathfrak{p}$ for all $\mathfrak{p} \in \bigcup_{i=1}^{d^{\prime}-j} \operatorname{Att}\left(H_{\mathfrak{m}}^{i}\left(M /\left(x_{1}, \ldots, x_{j}\right) M\right) \backslash\{\mathfrak{m}\}\right.$ for all $j=0, \ldots, k-1$. A sequence $\underline{x}=\left(x_{1}, \ldots, x_{k}\right)$ of elements in $\mathfrak{m}$ is called a permutable strict $f$-sequence of $M$ if it is a strict $\mathrm{f}$-sequence of $M$ in any order. It was proved in [CMN] that for each integer $k>0$, there always exists a strict f-sequence of $M$ of length $k$. Moreover, if $x$ is a strict f-element of $M$, then $0:_{M} x$ is of finite length. So, each strict f-sequence of $M$ containing at most $d^{\prime}$ elements is part of an s.o.p. of $M$. Note that strict f-sequences have been used (cf. [CMN]) to study the finiteness for attached primes of local cohomology modules, to study the polynomial property of the length of generalized fractions defined in $[\mathrm{SH}$, and to characterize the pseudo-generalized Cohen-Macaulay modules defined in [CN].

Lemma 2.2. The following statements are equivalent:

(i) Every s.o.p. of $M$ satisfies (MC).

(ii) Every permutable strict f-sequence s.o.p. of $M$ satisfies $(M C)$.

Proof. It is enough to prove (ii) $\Rightarrow(\mathrm{i})$. Let $\underline{x}=\left(x_{1}, \ldots, x_{d^{\prime}}\right)$ be an s.o.p. of $M$. Without loss of any generality we can assume that Ann $M=0$. We claim by induction on $k$ that there exists a permutable strict f-sequence $y_{1}, \ldots, y_{k}$ such that $\left(x_{1}, \ldots, x_{d^{\prime}}\right) R=\left(y_{1}, \ldots, y_{k}, x_{k+1}, \ldots, x_{d^{\prime}}\right) R$. Let $k=1$. Set $C_{1}=\bigcup_{i=1}^{d^{\prime}} \operatorname{Att}\left(H_{\mathfrak{m}}^{i}(M)\right)$. 
There exists by $\left[\mathrm{K}\right.$. Theorem 124] an element $a_{1} \in\left(x_{2}, \ldots, x_{d^{\prime}}\right) R$ such that $x_{1}+a_{1} \notin$ $\mathfrak{p}$ for all $\mathfrak{p} \in C_{1} \backslash\{\mathfrak{m}\}$. Let $y_{1}=x_{1}+a_{1}$. Then $\left(y_{1}, x_{2}, \ldots, x_{d^{\prime}}\right) R=\left(x_{1}, \ldots, x_{d^{\prime}}\right) R$ and $y_{1}$ is a permutable strict f-element of $M$. Let $1<k \leqslant d^{\prime}$ and assume that the claim is true for $k-1$. Set $C_{k}=\bigcup_{I \subseteq\{1, \ldots, k-1\}} \bigcup_{i=1}^{d^{\prime}} \operatorname{Att}\left(0:_{H_{\mathrm{m}}^{i}(M)} \sum_{j \in I} y_{j} R\right)$. Let $a_{k} \in\left(y_{1}, \ldots, y_{k-1}, x_{k+1}, \ldots, x_{d^{\prime}}\right) R$ such that $x_{k}+a_{k} \notin \mathfrak{p}$ for all $\mathfrak{p} \in C_{k} \backslash\{\mathfrak{m}\}$. Let $y_{k}=x_{k}+a_{k}$. It follows by the proof of [CMN, 4.2] that $\left(y_{1}, \ldots, y_{k}\right)$ is a permutable strict $\mathrm{f}$-sequence of $M$. By the induction hypothesis we have

$$
\left(x_{1}, \ldots, x_{d^{\prime}}\right) R=\left(y_{1}, \ldots, y_{k-1}, x_{k}, \ldots, x_{d^{\prime}}\right) R=\left(y_{1}, \ldots, y_{k}, x_{k+1}, \ldots, x_{d^{\prime}}\right) R,
$$

and the claim is proved. By the claim, there exists a permutable strict f-sequence $\underline{y}=\left(y_{1}, \ldots, y_{d^{\prime}}\right)$ of $M$ such that $\left(x_{1}, \ldots, x_{d^{\prime}}\right) R=\left(y_{1}, \ldots, y_{d^{\prime}}\right) R$. For each $i=$ $1, \ldots, d^{\prime}$ we write $x_{i}=\sum_{j=1}^{d^{\prime}} b_{i j} y_{j}$. Set $B=\left(b_{i j}\right)_{1 \leqslant i, j \leqslant d^{\prime}}$. Put $\delta=\operatorname{det} B$. Set $Q_{M}(\underline{y})=\cup_{t>0}\left(\left(y_{1}^{t+1}, \ldots, y_{d^{\prime}}^{t+1}\right) M:_{M} y_{1}^{t} \ldots y_{d^{\prime}}^{t}\right)$. For each $m+Q_{M}(\underline{y}) \in M / Q_{M}(\underline{y})$ we set $\delta\left(m+Q_{M}(\underline{y})\right)=\delta . m+Q_{M}(\underline{x})$, where $Q_{M}(\underline{x})$ is defined similarly. Then $\delta$ is a homomorphism from $M / Q_{M}(\underline{y})$ to $M / Q_{M}(\underline{x})$. This homomorphism is independent of the choice of the matrix $\bar{B}$, and it is called the determinantal map (cf. [St, 5.1.14]). By [CHL, 3.1], $\delta$ is injective. So, $\ell\left(M / Q_{M}(\underline{y})\right) \leqslant \ell\left(M / Q_{M}(\underline{x})\right)$. Since $\underline{y}$ satisfies $(\mathrm{MC})$, i.e. $\ell\left(M / Q_{M}(\underline{y})\right)>0$, it follows that $\bar{\ell}\left(M / Q_{M}(\underline{x})\right)>0$. Therefore $\underline{x}$ satisfies (MC).

Now we need to recall some knowledge of the theory of modules of generalized fractions [SZ1]. In this theory, for a given positive integer $k$, the so-called triangular subsets of $R^{k}$ play a similar role as multiplicatively closed subsets of $R$ do in the usual theory of localization of modules. Given a triangular subset $U$ of $R^{k}$, Sharp and Zakeri constructed an $R$-module $U^{-k} M$, and they called it the module of generalized fractions of $M$ with respect to $U$. In particular, the set

$$
\begin{aligned}
U(M)_{d^{\prime}+1}= & \left\{\left(y_{1}, \ldots, y_{d^{\prime}}, 1\right) \in R^{d^{\prime}+1}: \exists j, 0 \leqslant j \leqslant d^{\prime}, \text { such that }\left(y_{1}, \ldots, y_{j}\right)\right. \\
& \text { forms a subset of an s.o.p. of } \left.M \text { and } y_{j+1}=\ldots=y_{d^{\prime}}=1\right\}
\end{aligned}
$$

is a triangular subset of $R^{d^{\prime}+1}$. Let $\underline{x}=\left(x_{1}, \ldots, x_{d^{\prime}}\right)$ be an s.o.p. of $M$. Denote by $M\left(1 /\left(x_{1}, \ldots, x_{d^{\prime}}, 1\right)\right)$ the submodule $\left\{m /\left(x_{1}, \ldots, x_{d^{\prime}}, 1\right): m \in M\right\}$ of $U(M)_{d^{\prime}+1}^{-d^{\prime}-1} M$. Then the length of the submodule $M\left(1 /\left(x_{1}, \ldots, x_{d^{\prime}}, 1\right)\right)$ is always finite. Set

$$
q\left(x_{1}, \ldots, x_{d^{\prime}} ; M\right)=\ell\left(M\left(1 /\left(x_{1}, \ldots, x_{d^{\prime}}, 1\right)\right)\right) .
$$

Following Sharp-Hamieh $[\mathbf{S H}], q\left(x_{1}, \ldots, x_{d^{\prime}} ; M\right)$ is called the length of the generalized fraction $1 /\left(x_{1}, \ldots, x_{d^{\prime}}, 1\right)$ with respect to $M$. Note that the s.o.p. $\left(x_{1}, \ldots, x_{d^{\prime}}\right)$ of $M$ satisfies (MC) if and only if $q\left(x_{1}, \ldots, x_{d^{\prime}} ; M\right)>0$; cf. [SZ2].

From now on, we use the following notations from $[\mathrm{SH}]$ : Let $A$ be an Artinian $R$-module. The stability index of $A$, denoted by $s(A)$, is the least positive integer $s$ such that $\mathfrak{m}^{s} A=\mathfrak{m}^{n} A$ for all $n \geq s$. Denote by $\operatorname{Rl}(A)$ the length of $A / \mathfrak{m}^{s(A)} A$. Then $\mathrm{Rl}(A)$ is finite, and is called the residual length of $A$. It is clear that $\operatorname{Rl}(A)=0$ if and only if $\mathfrak{m} \notin \operatorname{Att} A$. Moreover, if $x \notin \mathfrak{p}$ for all $\mathfrak{p} \in \operatorname{Att} A \backslash\{\mathfrak{m}\}$, then $\ell(A / x A) \leqslant \operatorname{Rl}(A)$, and in this case, $\ell\left(A / x^{n} A\right)=\operatorname{Rl}(A)$ for all $n \geq s(A)$.

Theorem 2.3. Assume that $\underline{x}=\left(x_{1}, \ldots, x_{d^{\prime}}\right)$ is a strict f-sequence s.o.p. of $M$. Set $n_{\underline{x}}=\sum_{i=1}^{d^{\prime}-1} \operatorname{Rl}\left(H_{\mathfrak{m}}^{d^{\prime}-i}\left(M_{i-1}\right)\right)$, where $M_{0}=M, M_{i}=M /\left(x_{1}, \ldots, x_{i}\right) M$ for $i=1, \ldots, d^{\prime}-1$. If $e(\underline{x} ; M)>n_{\underline{x}}$, then $\underline{x}$ satisfies $(M C)$. 
Proof. Without loss of generality, we may assume that depth $M>0$. By $\underline{\mathrm{SH}}$, Proposition 2.2] we have the exact sequence

$$
0 \longrightarrow H_{\mathfrak{m}}^{d^{\prime}-1}(M) / x_{1} H_{\mathfrak{m}}^{d^{\prime}-1}(M) \longrightarrow U\left(M_{1}\right)_{d^{\prime}}^{-d^{\prime}} M_{1} \stackrel{\Psi_{d^{\prime}+1}}{\longrightarrow} U(M)_{d^{\prime}+1}^{-d^{\prime}-1} M,
$$

where $\Psi_{d^{\prime}+1}$ is defined by $\Psi_{d^{\prime}+1}\left(\bar{m} /\left(u_{2}, \ldots, u_{d^{\prime}}, 1\right)\right)=m /\left(x_{1}, u_{2}, \ldots, u_{d^{\prime}}, 1\right)$, for all $\bar{m} \in M_{1}$ and $\left(u_{2}, \ldots, u_{d^{\prime}}, 1\right) \in U\left(M_{1}\right)_{d^{\prime}}$. Consider $H_{\mathfrak{m}}^{d^{\prime}-1}(M) / x_{1} H_{\mathfrak{m}}^{d^{\prime}-1}(M)$ as a submodule of $U\left(M_{1}\right){\overline{d^{\prime}}}^{-d^{\prime}} M_{1}$. Then the above exact sequence implies the following exact sequence:

$$
\begin{aligned}
0 & \longrightarrow H_{\mathfrak{m}}^{d^{\prime}-1}(M) / x_{1} H_{\mathfrak{m}}^{d^{\prime}-1}(M) \cap M_{1}\left(1 /\left(x_{2}, \ldots, x_{d^{\prime}-1}, x_{d^{\prime}}, 1\right)\right) \\
& \longrightarrow M_{1}\left(1 /\left(x_{2}, \ldots, x_{d^{\prime}-1}, x_{d^{\prime}}, 1\right)\right) \stackrel{\Psi_{d^{\prime}+1}}{\longrightarrow} M\left(1 /\left(x_{1}, \ldots, x_{d^{\prime}-1}, x_{d^{\prime}}, 1\right)\right) \longrightarrow 0 .
\end{aligned}
$$

Let $s=s\left(H_{\mathfrak{m}}^{d^{\prime}-1}(M)\right)$, the stability index of $H_{\mathfrak{m}}^{d^{\prime}-1}(M)$. Since $x_{1}$ is a strict f-element of $M, \ell\left(H_{\mathfrak{m}}^{d^{\prime}-1}(M) / x_{1} H_{\mathfrak{m}}^{d^{\prime}-1}(M)\right)<\infty$. Therefore

$$
\begin{aligned}
\ell\left(H_{\mathfrak{m}}^{d^{\prime}-1}(M) / x_{1} H_{\mathfrak{m}}^{d^{\prime}-1}(M)\right. & \left.\cap M\left(1 /\left(x_{2}, \ldots, x_{d^{\prime}-1}, x_{d^{\prime}}, 1\right)\right)\right) \\
& \leqslant \ell\left(H_{\mathfrak{m}}^{d^{\prime}-1}(M) / x_{1} H_{\mathfrak{m}}^{d^{\prime}-1}(M)\right) \\
& \leqslant \ell\left(H_{\mathfrak{m}}^{d^{\prime}-1}(M) / \mathfrak{m}^{s} H_{\mathfrak{m}}^{d^{\prime}-1}(M)\right)=\operatorname{Rl}\left(H_{\mathfrak{m}}^{d^{\prime}-1}(M)\right) .
\end{aligned}
$$

Hence

$$
q\left(x_{1}, \ldots, x_{d^{\prime}-1}, x_{d^{\prime}} ; M\right) \geq q\left(x_{2}, \ldots, x_{d^{\prime}-1}, x_{d^{\prime}} ; M_{1}\right)-\operatorname{Rl}\left(H_{\mathfrak{m}}^{d^{\prime}-1}(M)\right) .
$$

Continuing this process we get

$$
\begin{aligned}
q\left(x_{1}, \ldots, x_{d^{\prime}-1}, x_{d^{\prime}} ; M\right) & \geq q\left(x_{2}, \ldots, x_{d^{\prime}-1}, x_{d^{\prime}} ; M_{1}\right)-\operatorname{Rl}\left(H_{\mathfrak{m}}^{d^{\prime}-1}(M)\right) \\
& \geq q\left(x_{3}, \ldots, x_{d^{\prime}-1}, x_{d^{\prime}} ; M_{2}\right)-\sum_{i=1}^{2} \operatorname{Rl}\left(H_{\mathfrak{m}}^{d^{\prime}-i}\left(M_{i-1}\right)\right) \\
& \geq \ldots \geq q\left(x_{d^{\prime}} ; M_{d^{\prime}-1}\right)-\sum_{i=1}^{d^{\prime}-1} \operatorname{Rl}\left(H_{\mathfrak{m}}^{d^{\prime}-i}\left(M_{i-1}\right)\right) \\
& =q\left(x_{d^{\prime}} ; M_{d^{\prime}-1}\right)-n_{\underline{x}} .
\end{aligned}
$$

Note that $\operatorname{dim} M_{d^{\prime}-1}=1$. Therefore $q\left(x_{d^{\prime}} ; M_{d^{\prime}-1}\right)=e\left(x_{d^{\prime}} ; M_{d^{\prime}-1}\right)$. Since $\underline{x}$ is an f-sequence of $M$, we get by the hypothesis that

$$
q\left(x_{d^{\prime}} ; M_{d^{\prime}-1}\right)=e\left(x_{d^{\prime}} ; M_{d^{\prime}-1}\right)=e(\underline{x} ; M)>n_{\underline{x}} .
$$

Hence $q\left(x_{1}, \ldots, x_{d^{\prime}} ; M\right)>0$, i.e. $\left(x_{1}, \ldots, x_{d^{\prime}}\right)$ satisfies (MC).

Note that the number $n_{\underline{x}}$ in Theorem 2.3 does not depend on $x_{d^{\prime}-1}, x_{d^{\prime}}$.

Corollary 2.4. Assume that $\underline{x}=\left(x_{1}, \ldots, x_{d^{\prime}}\right)$ is a strict f-sequence s.o.p. of $M$ and $n_{\underline{x}}$ is the integer defined as in Theorem 2.3. Then $\left(x_{1}, \ldots, x_{d^{\prime}-2}, x_{d^{\prime}-1}^{n}, x_{d^{\prime}}^{m}\right)$ satisfies $(M C)$ when $n m>n_{\underline{x}}$. In particular, $\left(x_{1}, \ldots, x_{d^{\prime}-1}, x_{d^{\prime}}^{n}\right)$ satisfies $(M C)$ when $n>n_{\underline{x}}$.

Proof. By [CMN, Cor. 3.5], $\left(x_{1}, \ldots, x_{d^{\prime}-2}, x_{d^{\prime}-1}^{n}, x_{d^{\prime}}^{m}\right)$ is a strict f-sequence of $M$ for all integers $n, m>0$. We have

$$
e\left(x_{1}, \ldots, x_{d^{\prime}-2}, x_{d^{\prime}-1}^{n}, x_{d^{\prime}}^{m} ; M\right)=n m e\left(x_{1}, \ldots, x_{d^{\prime}} ; M\right) \geq n m .
$$

So, by Theorem $2.3,\left(x_{1}, \ldots, x_{d^{\prime}-2}, x_{d^{\prime}-1}^{n}, x_{d^{\prime}}^{m}\right)$ satisfies (MC) for all $n m>n_{\underline{x}}$. 
Theorem 1.6 of Dutta D can be extended from rings to modules as follows.

Corollary 2.5. Given an s.o.p. $\underline{x}=\left(x_{1}, \ldots, x_{d^{\prime}}\right)$ of $M$, we can construct a strict f-sequence $\left(y_{1}, \ldots, y_{d^{\prime}-1}\right)$, for each $y_{i} \in\left(x_{1}, \ldots, x_{d^{\prime}}\right) R, i=1, \ldots, d^{\prime}-1$, such that $\left(x_{1}, \ldots, x_{d^{\prime}}\right) R=\left(y_{1}, \ldots, y_{d^{\prime}-1}, x_{d^{\prime}}\right) R$ and $\left(y_{1}, \ldots, y_{d^{\prime}-1}, x_{d^{\prime}}^{n}\right)$ satisfies $(M C)$ for $n \gg 0$.

Proof. Without loss of generality we can assume that Ann $M=0$. Let $\left(x_{1}, \ldots, x_{d^{\prime}}\right)$ be an s.o.p. of $M$. By using $\left[\mathrm{K}\right.$, Theorem 124], we can choose $\left(y_{1}, \ldots, y_{d^{\prime}-1}\right)$ such that

$$
\begin{aligned}
& y_{1}=x_{1}+a_{1}, \text { for some } a_{1} \in\left(x_{2}, \ldots, x_{d^{\prime}}\right) R, \\
& y_{2}=x_{2}+a_{2}, \text { for some } a_{2} \in\left(y_{1}, x_{3}, \ldots, x_{d^{\prime}}\right) R, \\
& \ldots \\
& y_{d^{\prime}-1}=x_{d^{\prime}-1}+a_{d^{\prime}-1}, \text { for some } a_{d^{\prime}-1} \in\left(y_{1}, \ldots, y_{d^{\prime}-2}, x_{d^{\prime}}\right) R,
\end{aligned}
$$

where $y_{k} \notin \mathfrak{p}$ for all $\mathfrak{p} \in \cup_{i=1}^{d^{\prime}-k+1} \operatorname{Att}\left(H_{\mathfrak{m}}^{i}\left(M /\left(y_{1}, \ldots, y_{k-1}\right) M\right)\right) \backslash\{\mathfrak{m}\}$, and all $k \leqslant$ $d^{\prime}-1$. Then $\left(y_{1}, \ldots, y_{d^{\prime}-1}, x_{d^{\prime}}\right)$ is a strict f-sequence of $M, y_{i} \in\left(x_{1}, \ldots, x_{d^{\prime}}\right) R$ for $i \leqslant$ $d^{\prime}$ and $\left(x_{1}, \ldots, x_{d^{\prime}}\right) M=\left(y_{1}, \ldots, y_{d^{\prime}-1}, x_{d^{\prime}}\right) M$. By Corollary $2.4,\left(y_{1}, \ldots, y_{d^{\prime}-1}, x_{d^{\prime}}^{n}\right)$ satisfies (MC) for $n \gg 0$.

Note that in general, (MC) is not valid for $M$ when $\operatorname{dim} M=2$, but it is always valid for the rings of dimension 2 . Therefore, to consider (MC) only for rings, we have the following statement which is stronger than that of Corollary 2.4.

Corollary 2.6. Let $\underline{x}=\left(x_{1}, \ldots, x_{d}\right)$ be a strict f-sequence of $R$. Set $R_{0}=R$ and $R_{i}=R /\left(x_{1}, \ldots, x_{i}\right) R$ for $i=1, \ldots, d-2$. Set $n_{\underline{x}}^{*}=\sum_{i=1}^{d-2} \mathrm{Rl}\left(H_{\mathfrak{m}}^{d-i}\left(R_{i-1}\right)\right)$. Then $\left(x_{1}, \ldots x_{d-2}, x_{d-1}^{m}, x_{d}^{n}\right)$ satisfies $(M C)$ when $m n>n_{\underline{x}}^{*}$. In particular, $\left(x_{1}, \ldots, x_{d^{\prime}-1}\right.$, $\left.x_{d^{\prime}}^{n}\right)$ satisfies $(M C)$ when $n>n_{\underline{x}}^{*}$.

Proof. We have as in the proof of Theorem 2.3 that

$$
\begin{aligned}
q\left(x_{1}, \ldots, x_{d} ; R\right) & \geq q\left(x_{d-1}, x_{d} ; R_{d-2}\right)-\sum_{i=1}^{d-2} \operatorname{Rl}\left(H_{\mathfrak{m}}^{d-i}\left(R_{i-1}\right)\right) \\
& =q\left(x_{d-1}, x_{d} ; R_{d-2}\right)-n_{\underline{x}}^{*}
\end{aligned}
$$

Since $\operatorname{dim} R_{d-2}=2$, (MC) is valid for $R_{d-2}$. Hence $q\left(x_{d-1}, x_{d} ; R_{d-2}\right)>0$. So, by CHL, Lemma 4.1(ii)] we have

$$
q\left(x_{d-1}^{n}, x_{d}^{m} ; R_{d-2}\right) \geq m n q\left(x_{d-1}, x_{d} ; R_{d-2}\right) \geq m n .
$$

Hence $q\left(x_{1}, \ldots x_{d-2}, x_{d-1}^{m}, x_{d}^{n} ; R\right)>0$, i.e. $\left(x_{1}, \ldots, x_{d-2}, x_{d-1}^{m}, x_{d}^{n}\right)$ satisfies (MC) when $m n>n_{\underline{x}}^{*}$.

Remark. The number $n_{x}^{*}$ in Corollary 2.6 does not depend on $x_{d-2}, x_{d-1}, x_{d}$. Moreover, Corollary 2.6 does not hold for modules. In fact, let $d \geq 2$ be an integer, let $R=k\left[\left[x_{1}, \ldots, x_{d}\right]\right]$ be the ring of powers series in $d$ variables over a field $k$ and let $M=\left(x_{1}, \ldots, x_{d}\right) R$. Then $M$ is a Buchsbaum module, $\left(x_{1}, \ldots, x_{d}\right)$ is a permutable strict f-sequence of $M$ and $\sum_{i=1}^{d-2} \operatorname{Rl}\left(H_{\mathfrak{m}}^{d-i}\left(M_{i-1}\right)\right)=0$, where $M_{0}=M$, $M_{i}=M /\left(x_{1}, \ldots, x_{i}\right) M$ for $i \leqslant d-2$. However, $\left(x_{1}, \ldots, x_{d}\right)$ does not satisfy (MC). 


\section{The strong uniform Bound for the Monomial Conjecture}

It has been shown that there always exists an integer $c>0$ depending only on $M$ such that $\left(x_{1}^{n_{1}}, \ldots, x_{d^{\prime}}^{n_{d^{\prime}}}\right)$ satisfies (MC) for all s.o.p. $\left(x_{1}, \ldots, x_{d^{\prime}}\right)$ of $M$ and all integers $n_{1}, \ldots, n_{d^{\prime}} \geq c$; cf. [CHL, Theorem 3.3]. This number $c$ is called a uniform bound for $(M C)$ of $M$. In this section, we study a stronger kind of the uniform bound as follows.

Definition 3.1. Let $c>0$ be an integer. $c$ is called a strong uniform bound for $(M C)$ of $M$ if $\left(x_{1}, \ldots, x_{d^{\prime}-1}, x_{d^{\prime}}^{n}\right)$ satisfies (MC) for all s.o.p. $\left(x_{1}, \ldots, x_{d^{\prime}}\right)$ of $M$ and all integers $n \geq c$.

By using strict f-sequences, we have the following reduction.

Lemma 3.2. Let $c>0$ be an integer. The following statements are equivalent:

(i) $c$ is a strong uniform bound for $(M C)$ of $M$.

(ii) For every strict f-sequence s.o.p. $\left(x_{1}, \ldots, x_{d^{\prime}}\right)$ of $M$, the s.o.p. $\left(x_{1}, \ldots\right.$, $\left.x_{d^{\prime}-1}, x_{d^{\prime}}^{n}\right)$ satisfies $(M C)$ for all integers $n \geq c$.

Proof. It is enough to prove (ii) $\Rightarrow(\mathrm{i})$. Let $\left(x_{1}, \ldots, x_{d^{\prime}}\right)$ be an s.o.p. of $M$ and $n \geq c$ an integer. Without any loss of generality, we can assume that Ann $M=0$. As in the proof of Corollary 2.5, we can choose a strict f-sequence $\left(y_{1}, \ldots, y_{d^{\prime}-1}\right)$ of $M$ such that

$$
\left(y_{1}, \ldots, y_{d^{\prime}-1}, x_{d^{\prime}}^{n}\right) R=\left(x_{1}, \ldots, x_{d^{\prime}-1}, x_{d^{\prime}}^{n}\right) R .
$$

It is clear that $\left(y_{1}, \ldots, y_{d^{\prime}-1}, x_{d^{\prime}}\right)$ is a strict f-sequence of $M$. So, $\left(y_{1}, \ldots, y_{d^{\prime}-1}, x_{d^{\prime}}^{n}\right)$ satisfies (MC) by the hypothesis (ii). By the same arguments as in the last part of the proof of Lemma 2.2 , it follows that $\left(x_{1}, \ldots, x_{d^{\prime}-1}, x_{d^{\prime}}^{n}\right)$ satisfies (MC).

For each s.o.p. $\underline{x}=\left(x_{1}, \ldots, x_{d^{\prime}}\right)$ of $M$ and each $d^{\prime}$-tuple of positive integers $\underline{n}=\left(n_{1}, \ldots, n_{d^{\prime}}\right)$, we set $\underline{x}(\underline{n})=\left(x_{1}^{n_{1}}, \ldots, x_{d^{\prime}}^{n_{d^{\prime}}}\right)$ and

$$
\begin{aligned}
& I(\underline{x}(\underline{n}) ; M)=\ell(M /(\underline{x}(\underline{n}) M))-e(\underline{x}(\underline{n}) ; M), \\
& J(\underline{x}(\underline{n}) ; M)=e(\underline{x}(\underline{n}) ; M)-\ell(M / Q(\underline{x}(\underline{n}) ; M)),
\end{aligned}
$$

where $Q(\underline{x} ; M)=\cup_{t>0}\left(x_{1}^{t+1}, \ldots, x_{d^{\prime}}^{t+1}\right) M:_{M} x_{1}^{t} \ldots x_{d^{\prime}}^{t}$. It is known that in general $I(\underline{x}(\underline{n}) ; M)$ and $J(\underline{x}(\underline{n}) ; M)$, considered as functions in $n_{1}, \ldots, n_{d^{\prime}}$, are not polynomials when $n_{1}, \ldots, n_{d^{\prime}} \gg 0$, but they always take non-negative values and are bounded above by polynomials. In particular, the least degree of all polynomials in $\underline{n}$ bounding above the function $I(\underline{x}(\underline{n}) ; M)(\operatorname{resp} . J(\underline{x}(\underline{n}) ; M))$ does not depend on the choice of $\underline{x}$ (cf. [C], $[\mathrm{CM}]$ ). This least degree is denoted by $p(M)$ (resp. $p f(M))$. Following N. T. Cuong $[\mathrm{C}, p(M)$ is called the polynomial type of $M$. If we stipulate that the degree of zero is $-\infty$, then $M$ is Cohen-Macaulay if and only if $p(M)=-\infty$, and $M$ is generalized Cohen-Macaulay if and only if $p(M) \leqslant 0$. In general, $p f(M) \leqslant p(M)$, and if $\operatorname{dim} M \geq 2$, then $p f(M) \leqslant \operatorname{dim} M-2$ (cf. [CM]).

The following fact has been proved in [CN, Corollary 3.5].

Lemma 3.3. If $p f(M) \leqslant 0$, then $M$ has a strong uniform bound for $(M C)$.

In the case $p f(M)>0$, we have the following result.

Theorem 3.4. Let $k \geq 0$ be an integer. Then there exists a finitely generated module $M$ over a regular local ring $R$ such that $p f(M)=k$ and $M$ does not have a strong uniform bound for $(M C)$. 
Proof. Let $d \geq k+2$ be an integer. Let $R=k\left[\left[x_{1}, \ldots, x_{d}\right]\right]$ be the ring of power series in $d$ variables over a field $k$. Let $\mathfrak{m}=\left(x_{1}, \ldots, x_{d}\right) R$ and let $M=\left(x_{1}, \ldots, x_{d-k}\right) R$. From the exact sequence

$$
0 \longrightarrow M \longrightarrow R \longrightarrow R / M \longrightarrow 0
$$

we can check that $H_{\mathfrak{m}}^{k+1}(M) \cong H_{\mathfrak{m}}^{k}(R / M)$ and $H_{\mathfrak{m}}^{i}(M)=0$ for all $i \neq k+1, i \neq d$. Note that

$$
\operatorname{dim} R / \operatorname{Ann}\left(H_{\mathfrak{m}}^{k+1}(M)\right)=\operatorname{dim} R / \operatorname{Ann}\left(H_{\mathfrak{m}}^{k}(R / M)\right)=k .
$$

So, $p(M)=\max _{i<d} \operatorname{dim} R / \operatorname{Ann}\left(H_{\mathfrak{m}}^{i}(M)\right)=k$ by [C, Theorem 1.2], where $p(M)$ is the polynomial type of $M$. Since depth $M=k+1>k=p(M)$, we get by CM, Proposition 3.5, (ii)] that $p f(M)=k$. For the rest of the statement, it is clear that $\left(x_{1}, \ldots, x_{d}\right)$ is an s.o.p. of $M$. Since $k>0$, we can easily check that $\left(x_{1}, \ldots, x_{d-1}, x_{d}^{n}\right)$ does not satisfy (MC) for all $n>0$. So $M$ does not have a strong uniform bound for $(\mathrm{MC})$.

Remark. It would be of interest to prove a weaker statement of Monomial Conjecture: Every local ring has a strong uniform bound for $(M C)$.

\section{A characterization of Cohen-Macaulay CANonical modules}

The notion of Cohen-Macaulay canonical modules was introduced by P. Schenzel Sc] as follows.

Definition 4.1. Assume that $R$ admits a dualizing complex. $M$ is called a CohenMacaulay canonical module if the canonical module $K(M)$ of $M$ is Cohen-Macaulay.

It is clear that if $M$ is a Cohen-Macaulay canonical module with $\operatorname{dim} M=d$, then the canonical module $K(M)$ of $M$ is a maximal Cohen-Macaulay module. In this case, $(\mathrm{MC})$ is valid for $R$. Therefore it is of interest to find conditions for a module to be Cohen-Macaulay canonical. Schenzel [Sc, Theorem 1.1] gave a characterization of Cohen-Macaulay canonical domains in term of a so-called birational Macaulayfication. There is also a characterization for unmixed Cohen-Macaulay canonical modules $M$ of depth $M=d^{\prime}-1$ in terms of the Cohen-Macaulayness of the $d^{\prime}-1^{\text {th }}$ deficiency module $K^{d^{\prime}-1}(M)$ of $M$; cf. [Sc]. However, as far as the author knows, there is no characterization of Cohen-Macaulay canonical modules $M$ in terms of intrinsic data of $M$. Such characterization is given below.

From now on, for each finitely generated $R$-module $N$ with $\operatorname{dim} N=d^{\prime}$ and each strict f-sequence $\underline{x}=\left(x_{1}, \ldots, x_{d^{\prime}}\right)$ of $N$, we set $N_{\underline{x}, 0}=N$ and $N_{\underline{x}, i}=N /\left(x_{1}, \ldots, x_{i}\right) N$ for $i=1, \ldots, d^{\prime}-2$. When $R$ admits a dualizing complex, we denote by $K^{i}(N)$ the $i^{t h}$ deficiency module of $N$ for $i=1, \ldots, d^{\prime}-1$, and $K(N)=K^{d^{\prime}}(N)$ the canonical module of $N$; see [Sc, Definition 2.1].

Theorem 4.2. Assume that $R$ admits a dualizing complex. The following statements are equivalent:

(i) $M$ is a Cohen-Macaulay canonical module.

(ii) $\sum_{i=1}^{d^{\prime}-2} \operatorname{Rl}\left(H_{\mathfrak{m}}^{d^{\prime}-i}\left(M_{\underline{x}, i-1}\right)\right)=0$ for all strict f-sequences s.o.p. $\underline{x}$ of $M$.

(iii) $\sum_{i=1}^{d^{\prime}-2} \operatorname{Rl}\left(H_{\mathfrak{m}}^{d^{\prime}-i}\left(M_{\underline{x}}, i-1\right)\right)=0$ for a strict $f$-sequence s.o.p. $\underline{x}$ of $M$. 
Proof. (i) $\Rightarrow$ (ii). We prove by induction on $d^{\prime}$. There is nothing to prove for the case $d^{\prime} \leqslant 2$. Let $d^{\prime} \geq 3$. Let $\underline{x}=\left(x_{1}, \ldots, x_{d^{\prime}}\right)$ be an arbitrary strict f-sequence of $M$. Then $\ell\left(0:_{M} x_{1}\right)<\infty$. Therefore, from the exact sequence

$$
0 \longrightarrow M / 0:_{M} x_{1} \stackrel{. x_{1}}{\longrightarrow} M \longrightarrow M / x_{1} M \longrightarrow 0
$$

we get the exact sequences

$$
0 \longrightarrow K^{i+1}(M) / x_{1} K^{i+1}(M) \longrightarrow K^{i}\left(M / x_{1} M\right) \longrightarrow 0:_{K^{i}(M)} x_{1} \longrightarrow 0
$$

for $i=0,1, \ldots, d^{\prime}-1$. Since $K(M)$ is Cohen-Macaulay by the assumption (i) and $x_{1}$ is a regular element of $K(M)$, it follows that $K(M) / x_{1} K(M)$ is Cohen-Macaulay. Moreover, $\operatorname{since} \operatorname{depth}\left(K\left(M / x_{1} M\right)\right)>0$, we have $H_{\mathfrak{m}}^{0}\left(K\left(M / x_{1} M\right)\right)=0$. So, we get by the exact sequence $(*)$ with respect to $i=d^{\prime}-1$ that $H_{\mathfrak{m}}^{0}\left(0:_{K^{d^{\prime}-1}(M)} x_{1}\right)=0$. Since $x_{1}$ is a strict f-element of $M$, we can check that $\ell\left(0:_{K^{d^{\prime}-1}(M)} x_{1}\right)<\infty$. Hence

$$
0:_{K^{d^{\prime}-1}(M)} x_{1}=H_{\mathfrak{m}}^{0}\left(0:_{K^{d^{\prime}-1}(M)} x_{1}\right)=0 .
$$

So $K(M) / x_{1} K(M) \cong K\left(M / x_{1} M\right)$ by the exact sequence $(*)$ with respect to $i=$ $d^{\prime}-1$. Hence $K\left(M / x_{1} M\right)$ is Cohen-Macaulay, i.e. $M / x_{1} M$ is Cohen-Macaulay canonical. Since $\underline{x}^{\prime}=\left(x_{2}, \ldots, x_{d^{\prime}}\right)$ is a strict f-sequence of $M / x_{1} M$, we get by the induction hypothesis that

$$
\sum_{i=1}^{\left(d^{\prime}-1\right)-2} \operatorname{Rl}\left(H_{\mathfrak{m}}^{\left(d^{\prime}-1\right)-i}\left(\left(M / x_{1} M\right)_{\underline{x}^{\prime}, i-1}\right)\right)=0,
$$

i.e.

$$
\sum_{i=2}^{d^{\prime}-2} \operatorname{Rl}\left(H_{\mathfrak{m}}^{d^{\prime}-i}\left(M_{\underline{x}, i-1}\right)\right)=0 .
$$

Since $0: K_{K^{d^{\prime}-1}(M)} x_{1}=0$, we obtain $\mathfrak{m} \notin \operatorname{Ass} K^{d^{\prime}-1}(M)$. Hence $\operatorname{Rl}\left(H_{\mathfrak{m}}^{d^{\prime}-1}(M)\right)=0$ and hence $\sum_{i=1}^{d^{\prime}-2} \operatorname{Rl}\left(H_{\mathfrak{m}}^{d^{\prime}-i}\left(M_{\underline{x}, i-1}\right)\right)=0$.

(ii) $\Rightarrow$ (iii) is trivial.

(iii) $\Rightarrow$ (i). Let $\underline{x}=\left(x_{1}, \ldots, x_{d^{\prime}}\right)$ be a strict f-sequence of $M$ which satisfies the condition as in (iii). We prove statement (i) by induction on $d^{\prime}$. The case $d^{\prime} \leqslant 2$ is already true. Let $d^{\prime} \geq 3$. Note that $\underline{x}^{\prime}=\left(x_{2}, \ldots, x_{d^{\prime}}\right)$ is a strict f-sequence of $M / x_{1} M$. Moreover, by (iii),

$$
\sum_{i=2}^{d^{\prime}-2} \operatorname{Rl}\left(H_{\mathfrak{m}}^{d^{\prime}-i}\left(M_{\underline{x}, i-1}\right)\right)=0
$$

i.e.

$$
\sum_{i=1}^{\left(d^{\prime}-1\right)-2} \operatorname{Rl}\left(H_{\mathfrak{m}}^{\left(d^{\prime}-1\right)-i}\left(\left(M / x_{1} M\right)_{\underline{x}^{\prime}, i-1}\right)\right)=0 .
$$

So we can apply the induction hypothesis on $M / x_{1} M$, and we get that $K\left(M / x_{1} M\right)$ is Cohen-Macaulay. It follows by (iii) that $\operatorname{Rl}\left(H_{\mathfrak{m}}^{d^{\prime}-1}(M)\right)=0$. Therefore $\mathfrak{m} \notin$ $\operatorname{Att}\left(H_{\mathfrak{m}}^{d^{\prime}-1}(M)\right)$. Moreover, $x_{1} \notin \mathfrak{p}$ for all $\mathfrak{p} \in \operatorname{Att} H_{\mathfrak{m}}^{d^{\prime}}-1(M) \backslash\{\mathfrak{m}\}$. These facts imply that $0::_{K_{M}^{d^{\prime}-1}} x_{1}=0$. So, we have by the exact sequence $(*)$ with respect to $i=d^{\prime}-1$ that $K(M) / x_{1} K(M) \cong K\left(M / x_{1} M\right)$. Since $K\left(M / x_{1} M\right)$ is Cohen-Macaulay and $x_{1}$ is a regular element of $K(M)$, we get that $K(M)$ is Cohen-Macaulay, i.e. $M$ is Cohen-Macaulay canonical. 
The following result gives a sufficient condition for a ring $R$ to satisfy (MC).

Corollary 4.3. If there exists a finitely generated $R$-module $M$ with $\operatorname{dim} M=d$, and a strict f-sequence s.o.p. $\underline{x}$ of $M$ such that $\mathfrak{m} \notin \operatorname{Att} H_{\mathfrak{m}}^{d-i}\left(M_{\underline{x}, i-1}\right)$ for all $i=1, \ldots, d-2$, then $(M C)$ is valid for $R$.

Proof. It follows by the assumption that $\sum_{i=1}^{d-2} \mathrm{Rl}\left(H_{\mathfrak{m}}^{d-i}\left(M_{\underline{x}, i-1}\right)\right)=0$. Therefore $\sum_{i=1}^{d-2} \operatorname{Rl}\left(H_{\mathfrak{m} \widehat{R}}^{d-i}\left(\widehat{M}_{\underline{x}, i-1}\right)\right)=0$. So, $\widehat{M}$ is Cohen-Macaulay canonical by Theorem 4.2 . Hence (MC) is valid for $\widehat{R}$ and for $R$.

The following result has been proved by Schenzel [Sc, Theorem 4.3] by using some knowledge on spectral sequences. Here we use strict f-sequences to give an elementary proof.

Corollary 4.4. Suppose that $R$ has a dualizing complex. If $\operatorname{depth}\left(K^{i}(M)\right) \geq i-1$ for all $2 \leqslant i<d^{\prime}$, then $M$ is Cohen-Macaulay canonical.

Proof. Let $\underline{x}=\left(x_{1}, \ldots, x_{d^{\prime}}\right)$ be a strict f-sequence of $M$. By Theorem 4.2, it is enough to prove $\sum_{i=1}^{d-2} \operatorname{Rl}\left(H_{\mathfrak{m}}^{d-i}\left(M_{\underline{x}, i-1}\right)\right)=0$. We prove this by induction on $d^{\prime}$. The case $d^{\prime} \leqslant 2$ is trivial. Let $d^{\prime} \geq 3$. As in the proof of Theorem 4.2, we have the exact sequences

$$
0 \longrightarrow K^{i+1}(M) / x_{1} K^{i+1}(M) \longrightarrow K^{i}\left(M / x_{1} M\right) \longrightarrow 0:_{K^{i}(M)} x_{1} \longrightarrow 0
$$

for $i=0,1, \ldots, d^{\prime}-1$. Let $2 \leqslant i \leqslant d^{\prime}-1$. Since $\operatorname{depth}\left(K^{i}(M)\right)>0$, we have $0: K_{K^{i}(M)} x_{1}=0$. So, by the above exact sequences, $\operatorname{depth} K^{i}\left(M / x_{1} M\right) \geq i-1$ for all $2 \leqslant i<d^{\prime}-1$. Therefore $\sum_{i=1}^{\left(d^{\prime}-1\right)-2} \operatorname{Rl}\left(H_{\mathfrak{m}}^{\left(d^{\prime}-1\right)-i}\left(\left(M / x_{1} M\right)_{\underline{x}^{\prime}, i-1}\right)\right)=0$ by the induction hypothesis, where $\underline{x}^{\prime}=\left(x_{2}, \ldots, x_{d^{\prime}}\right)$. So, $\sum_{i=2}^{d^{\prime}-2} \operatorname{Rl}\left(H_{\mathfrak{m}}^{d^{\prime}-i}\left(M_{\underline{x}, i-1}\right)\right)=0$. Since $\operatorname{depth}\left(K^{d^{\prime}-1}(M)\right)>0$, we get $\operatorname{Rl}\left(H_{\mathfrak{m}}^{d^{\prime}-1}(M)\right)=0$, and the result follows.

By Theorem 4.2 and by induction on $d^{\prime}$, we can easily prove the following result. Note that this result has been proved in $[\mathrm{Sc}$, Theorem 4.6, (iii)] in case $M$ is umixed.

Corollary 4.5. Assume that $R$ admits a dualizing complex and $\operatorname{depth} M=d^{\prime}-1$. Then $M$ is Cohen-Macaulay canonical if and only if $K^{d^{\prime}-1}(M)$ is Cohen-Macaulay of dimension at least $d^{\prime}-2$.

\section{ACKNOWLEDGMENT}

The author is grateful to Professor N. T. Cuong for suggestions concerning the results in Section 3. She is also thankful to ICTP Trieste Italy for the financial support.

\section{REFERENCES}

[C] N. T. Cuong, On the dimension of non Cohen-Macaulay locus of local rings admitting dualizing complexes, Math. Proc. Camb. Soc., 109 (1991), 479-488. MR.1094747(92b:13034)

[CM] N. T. Cuong and N. D. Minh, Lengths of generalized fractions of modules having small polynomial type, Math. Proc. Camb. Phil. Soc., 128 (2000), 269-282. MR1735305 (2001c:13036)

[CHL] N. T. Cuong, N. T. Hoa and N. T. H. Loan, On certain length functions associated to a system of parameters in local rings, Vietnam J. Math., (3) 27 (1999), 259-272. MR 1811339 (2002d:13031)

[CN] N. T. Cuong and L. T. Nhan, On pseudo Cohen-Macaulay and pseudo generalized CohenMacaulay modules, J. Algebra, 267 (2003), 156-177. MR.1993472(2004f:13012) 
[CMN] N. T. Cuong, M. Morales and L. T. Nhan, The finiteness of certain sets of attached prime ideals and the length of generalized fractions, J. Pure Appl. Algebra, (1-3) 189 (2004), 109-121. MR2038567 (2004m:13046)

[D] S. P. Dutta, A note on the monomial conjecture, Trans. AMS, (7) 350 (1998), 2871-2878. MR 1466948 (98i:13047)

[Go] S. Goto, On the associated graded rings of parameter ideals in Buchsbaum rings, $J$. Algebra, 85 (1983), 490-534. MR0725097 (85d:13032)

[H1] M. Hochster, Contracted ideals from integral extensions of regular rings, Nagoya Math. J., 51 (1973), 25-43. MR0349656(50:2149)

[H2] M. Hochster, Canonical elements in local cohomology modules and the direct summand conjecture, J. Algebra, 84 (1983), 503-553. MR0723406 (85j:13021)

[K] I. Kaplansky, Commutative Rings, Allyn and Bacon, Boston, 1970. MR 0254021 (40:7234)

[Mac] I. G. Macdonald, Secondary representation of modules over a commutative ring, Symposia Mathematica, 11 (1973), 23-43. MR0342506 (49:7252)

[Sc] P. Schenzel, On birational Macaulayfications and Cohen-Macaulay canonical modules, $J$. Algebra, 275 (2004), 751-770. MR.2052635(2005i:13017)

[SH] R. Y. Sharp and M. A. Hamieh, Lengths of certain generalized fractions, J. Pure Appl. Algebra, 38 (1985), 323-336. MR0814188 (87c:13031)

[SZ1] R. Y. Sharp and H. Zakeri, Modules of generalized fractions, Mathematika, 29 (1982), 32-41. MR0673503 (84a:13008)

[SZ2] R. Y. Sharp and H. Zakeri, Generalized fractions and the monomial conjecture, J. Algebra, (2) 92 (1985), 380-388. MR0778456 (86c:13013)

[St] J. R. Strooker, Homological questions in local algebra, London Math. Lecture Notes Series, Vol. 145, Cambridge University Press, 1990. MR1074178 (91m:13013)

[SS] J. R. Strooker and J. Stückrad, Monomial conjecture and complete intersections, Manuscripta Math., 79 (1993), 153-159. MR1216771 (94c:13026)

Department of Mathematics, Thai Nguyen Pedagogical University, Thai Nguyen, VIETNAM

E-mail address: trtrnhan@yahoo.com 